\title{
Joint Channel Sensing and Power Control Scheme for Cognitive Radio Wireless Sensor Networks
}

\author{
Ziqiang Feng, Ian Wassell \\ Computer Laboratory \\ University of Cambridge, UK \\ Email: $\{$ zf232, ijw24\}@cam.ac.uk
}

\begin{abstract}
In this paper, we investigate channel sensing and power control problems in a cluster-based cognitive radio wireless sensor network (CRWSN). We first propose three channel sensing algorithms to solve the channel sensing problem including availability constraints. Then a joint channel sensing and power control scheme (JCSPCS) is proposed to solve the power control problem including quality of service $(\mathrm{QoS})$ constraints. We prove that all our algorithms are probably approximately correct (PAC) algorithms that can correctly identify a bad channel with high probability. We show that our algorithms are suitable for QoSconstrained applications as they all terminate in a finite time with a finite error rate. The performance of our channel sensing algorithms and the joint channel sensing and power control scheme is shown in the simulation results.
\end{abstract}

\section{INTRODUCTION}

In a traditional wireless sensor network (WSN), sensors usually operate in the Industrial, Scientific and Medical (ISM) unlicensed bands and have a limited energy supply. However, the development of the internet of things (IoT) and the high demand in the unlicensed bands has given rise to the problem of coexistence of heterogeneous systems. It is thus challenging to deploy WSN in busy unlicensed bands, especially for QoSconstrained applications. On the other hand, most licensed spectrum is underutilized and stays unoccupied for most of the time [1]. Cognitive radio (CR) is thus considered as a method to improve the spectrum efficiency for QoS-constrained applications in a WSN [2].

CR allows secondary users (unlicensed users) to opportunistically access the vacant channels of primary users (licensed users). However, secondary users must not interfere with the primary users. Since the channel availability statistics are generally unknown to the secondary users, they have to sense the available vacant channels before transmitting any data. The problem of channel sensing and access in cognitive radio networks has been widely investigated. Some of the literature has modeled the channel sensing problem as a multiarmed bandit problem [3]. In [4], the author models the opportunistic spectrum access problem as a restless multiarmed bandit problem and propose a regenerative cycle algorithm to track the best channel, i.e., that with the best availability statistics. A traditional exploration and exploitation problem is thus investigated. In [5], a decentralized online learning algorithm is proposed to find available channels for multiple users. The algorithm is designed based on the well known Upper Confidence Bound (UCB) [3] policy that yields logarithmic regret over time without requiring any information on channel availability statistics.
Cognitive wireless sensors can only afford to sense part of the spectrum at a time. Cooperative sensing is thus needed for joint channel sensing. In addition, the detected available channels in a CRWSN must be coordinated to avoid collisions. Therefore, a cluster-based CRWSN architecture is proposed. For a QoS-constrained application in a CRWSN, a channel must be assigned as soon as possible if a node makes a data transmission request. Instead of tracking the good channels, sensor nodes in our model sense their pre-assigned part of the spectrum and only eliminate the bad channels, but with high confidence. We show that our algorithms can always assign an available channel to a sensor node that has a data transmission request. Furthermore, our channel sensing algorithms terminate in a finite time and with a limited error rate. In order to maximize the lifetime of each sensor nodes and meet the QoS constraints such as maximum delay and minimum data rate, a joint channel sensing and power control scheme (JCSPCS) is proposed in this paper that can improve the energy efficiency of sensors in the network without violating the QoS contraints.

\section{System Model AND Problem Formulation}

Consider the cluster-based CRWSN in Fig. 1 with $K$ licensed orthogonal channels allocated to PUs for slotted transmission. Cognitive radio wireless sensors can transmit their data to the corresponding cluster head through licensed channels that are sensed idle in each time slot. In each cluster, each channel is pre-assigned to a sensor for channel sensing. Let $\mathcal{S}=\left\{S_{1}, S_{2}, \ldots, S_{K}\right\}$ and $\mathcal{C}=\left\{C_{1}, C_{2}, \ldots, C_{K}\right\}$ denote the set of sensors and the corresponding pre-assigned channels in one cluster respectively. We use $\mathcal{T}=\left\{T_{1}, T_{2}, \ldots\right\}$ to represent the set of time slots in the network. At the beginning of each time slot, the wireless sensors in $\mathcal{S}$ sense their preassigned channels and report the sensing results as well as their data transmission request (if any) to the cluster head via a control channel. The cluster head then notifies the sensors with their assigned channels for transmission. Note that for each sensor with a data transmission request, the assigned channel for transmission is selected from the available channels and does not necessarily have to be the same as the pre-assigned one.

For sensor $S_{i}$ sensing $C_{i}$ at time slot $T_{j}$, we use a random variable $\theta_{i, j}$ to represent whether $C_{i}$ is idle $\left(\theta_{i, j}=1\right)$ or not $\left(\theta_{i, j}=0\right)$. We also use $\omega_{i, j}$ to represent the data transmission request of sensor $S_{i}$ at time slot $T_{j}$, where $\omega_{i, j}=1$ represents that $S_{i}$ has data to send and $\omega_{i, j}=0$ otherwise. A message containing $\left(\theta_{i, j}, \omega_{i, j}\right)$ is sent to the cluster head via a control channel after channel sensing. 


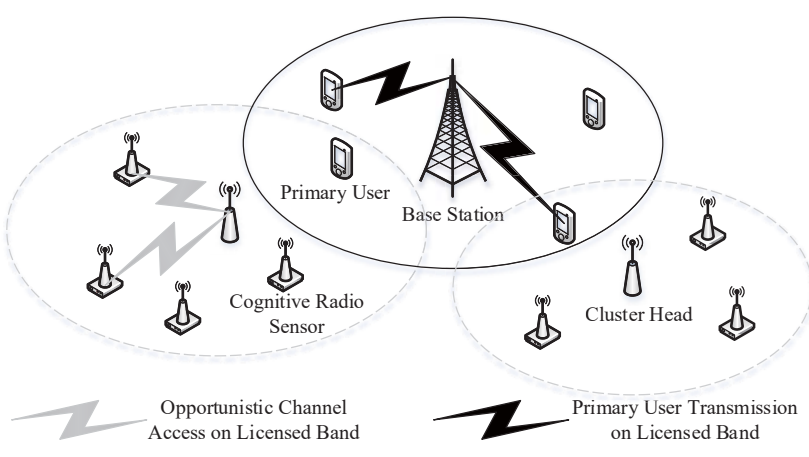

Fig. 1: Cluster-based cognitive radio wireless sensor network

\section{A. Channel Sensing with Availability Constraints}

In the CRWSN, sensors are only allowed to access the idle channels. The availability of the licensed channels is determined by the primary users' behavior. Therefore, we can improve the channel sensing efficiency if we can predict the channel availability by utilizing the channel sensing history. As each channel only has two states (busy or idle), we assume that the availability of channels in $\mathcal{C}$ follows independent identical Bernoulli distributions over different time slots with parameters $\mu=\left\{\mu_{1}, \mu_{2}, \ldots, \mu_{K}\right\}$ which are unknown to the sensors. The empirical mean availability of channel $C_{i}$ after the channel sensing in time slot $T_{n}$ is expressed as

$$
\hat{\mu}_{i, n}=\frac{1}{n} \sum_{j=1}^{n} \theta_{i, j} .
$$

Assuming that we have a channel availability requirement $\mu^{*}$ for all channels, we define the good channel set as $C_{G}=\left\{C_{i} \in \mathcal{C}: \mu_{i} \geq \mu^{*}\right\}$ and the bad channel set as $C_{B}=$ $\left\{C_{i} \in \mathcal{C}: \mu_{i}<\mu^{*}\right\}$ respectively. Sensors have to estimate the mean availability via (1). We assume that sensor $S_{i}$ use energy $\eta_{i} E_{i}$ for channel sensing where $E_{i}$ is the total energy of $S_{i}$ and $\eta_{i} \in[0,1]$ is the fraction of energy used for channel sensing. For simplicity and without loss of generality, we assume that all sensors have the same total energy $E$ and ratio $\eta$.

With the sensing energy constraint $\eta E$, we use an arbitrary algorithm $\rho \in \Omega$ to classify the channels into an empirical good set $\left(\hat{C}_{G}(\rho, \eta)\right)$ and bad set $\left(\hat{C}_{B}(\rho, \eta)\right)$ based on the past channel sensing results where $\Omega$ is the set of algorithms we use. We define the set of misclassified channels with algorithm $\rho$ and energy ratio $\eta$ as $C_{E}(\rho, \eta)=\left\{C_{i} \in \hat{C}_{G}(\rho, \eta): \mu_{i}<\mu^{*}\right\}$. The error rate is thus defined as $\varepsilon(\rho, \eta)=\frac{\left|C_{E}(\rho, \eta)\right|}{|\mathcal{C}|}$.

We aim at finding an algorithm $\rho^{*} \in \Omega$ that can find the good and bad channels in a limited time with high confidence and with a minimum error rate which is expressed as

$$
\begin{array}{r}
\rho^{*}=\underset{\rho \in \Omega}{\arg \min } \varepsilon(\rho, \eta)=\underset{\rho \in \Omega}{\arg \min }\left(\frac{\left|C_{E}(\rho, \eta)\right|}{K}\right), \\
\text { s.t. } \quad\left(P_{i, c}+P_{i, s}\right) \alpha T N_{i, \max } \leq \eta E, \forall i \in\{1,2 \ldots, K\} .
\end{array}
$$

where $P_{i, c}$ is the circuit power consumption of $S_{i}, P_{i, s}$ is the channel sensing power consumption of $S_{i}, T$ is the length of each time slot, $\alpha$ is the fraction of time used for channel sensing in each time slot and $N_{i, \max }$ is the maximum number of time slots for $S_{i}$ to spend on channel sensing. According to (2), the maximum number of time slots $N$ is expressed as

$$
N=\min _{i} N_{i, \max }=\min _{i}\left(\left\lfloor\frac{\eta E}{\alpha T\left(P_{i, c}+P_{i, s}\right)}\right\rfloor\right) .
$$

\section{B. Power Control with QoS Constraints}

Although increasing the transmission power level generally increases the data rate, it also decreases the lifetime of the sensor node. In this paper, we aim at finding the transmission power level that can maximize the total number of transmitted bits for QoS-constrained applications. We assume that all sensors have the same set of transmission power levels denoted by $P=\left\{P_{1}, P_{2}, \ldots, P_{M}\right\}$ in ascending order.

We use $R_{i, j, k}=f_{i}\left(P_{j}, C_{k}\right)$ to denote the maximum data rate of sensor $S_{i}$ with power $P_{j}$ at channel $C_{k}$. For simplicity, we assume that the maximum data rate of sensor $S_{i} \in \mathcal{S}$ with power $P_{j}$ at channel $C_{k}$ is a logarithmic function denoted as $f_{i}\left(P_{j}, C_{k}\right)=W \log _{2}\left(1+P_{j} g_{i, k}\right)$ where $W$ is the channel bandwidth and $g_{i, k}$ is the instantaneous channel gain to noise ratio of $C_{k}$ for $S_{i}$. The total number of transmitted bits for sensor $S_{i}$ with transmission power $P_{j}$ at channel $C_{k}$ is expressed as

$$
L_{i, j, k}=\frac{(1-\eta) E W \log _{2}\left(1+P_{j} g_{i, k}\right)}{\left(P_{j}+P_{i, c}\right)} .
$$

Assuming that $S_{i}$ has a minimum data rate requirement denoted by $R_{i}^{*}$, the optimal transmission power for $C_{k}$ that can maximize the total number of transmitted bits is expressed as

$$
\begin{aligned}
& P_{i, k}^{*}=\underset{P_{j} \in P}{\arg \max } L_{i, j, k}=\underset{P_{j} \in P}{\arg \max }\left(\frac{R_{i, j, k}(1-\eta) E}{P_{j}+P_{i, c}}\right), \\
& \text { s.t. } \quad R_{i, j, k} \geq R_{i}^{*}, \forall S_{i} \in S, C_{k} \in C ; \quad \eta \geq \eta^{*} ;
\end{aligned}
$$

where $\eta^{*}$ is the minimum energy ratio for reliable channel sensing. We use $L_{i, k}^{*}$ to denote the optimal total number of transmitted bits for $S_{i}$ on $C_{k}$ using power $P_{i, k}^{*}$. Let $\lambda_{i, k}=1$ if $C_{k}$ is accessed by $S_{i}$ and $\lambda_{i, k}=0$ otherwise. The maximum number of transmitted bits in the each cluster is expressed as

$$
\begin{aligned}
& L^{*}=\max \sum_{i=1}^{K} \sum_{k=1}^{K} \lambda_{i, k} L_{i, k}^{*}, \\
& \text { s.t. } \quad \sum_{k=1}^{m} \lambda_{i, k}=1, \forall i ; \sum_{i=1}^{n} \lambda_{i, k} \leq 1, \forall k .
\end{aligned}
$$

\section{ALGORITHMS}

In this section we introduce algorithms to solve the channel sensing problem with availability and power constraints.

\section{A. Passive Rejection Algorithm}

Under a passive rejection algorithm, sensor $S_{i}$ always senses its corresponding channel $C_{i}$ until it has high confidence that $\mu_{i}$ is less than the minimum channel availability requirement $\mu^{*}$. We first propose a simple reject algorithm (SRA) denoted by $\rho_{S R A}$ as detailed in Algorithm 1. SRA is an $\varepsilon$-PAC algorithm that can correctly identify a bad channel with probability at least $1-\varepsilon$. An algorithm is an $\varepsilon$-probably approximately correct (PAC) algorithm if it outputs the correct result with probability at least $1-\varepsilon$. The proof is omitted due to the space limit. 


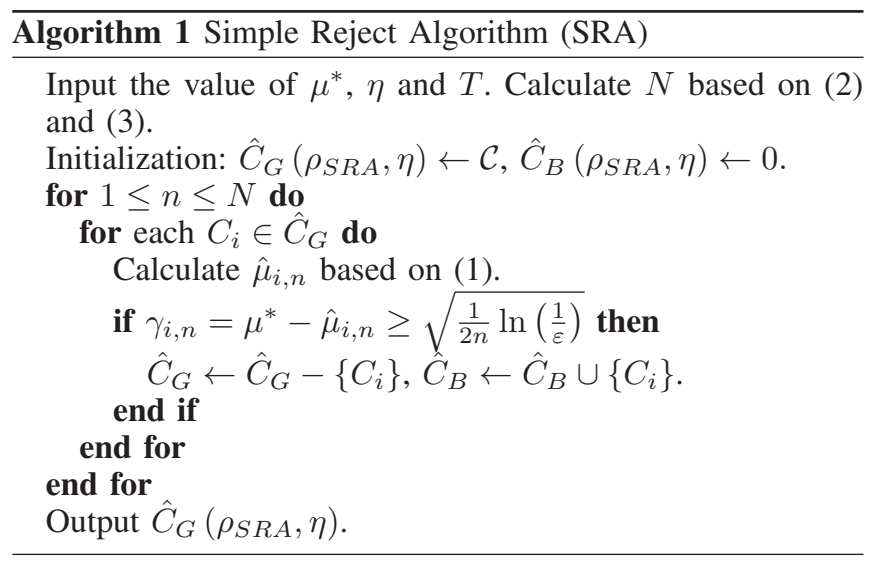

We also propose an improved reject algorithm (IRA) denoted by $\rho_{I R A}$ as detailed in Algorithm 2, which is also an $\varepsilon$-PAC algorithm.

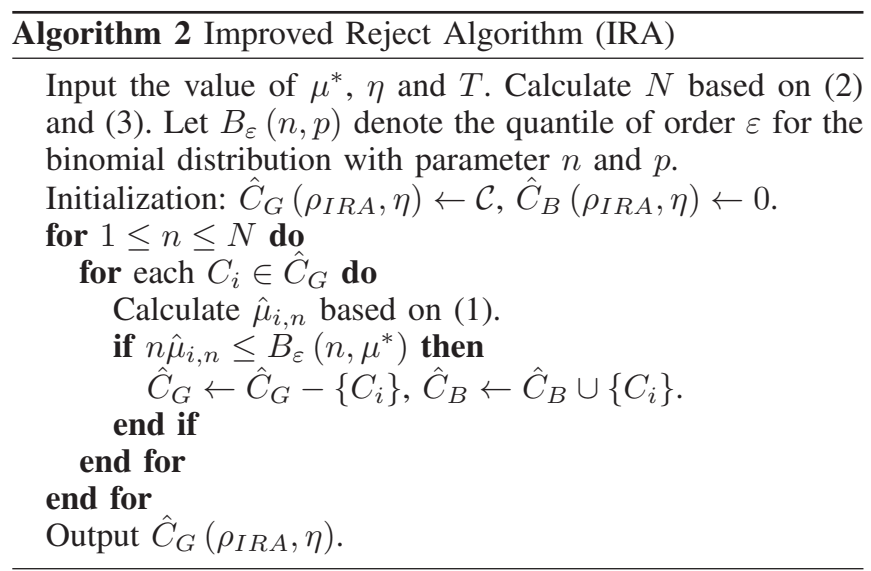

\section{B. Active Elimination Algorithm}

Although passive rejection algorithms can identify bad channels with high confidence, classifying channels with mean value close to $\mu^{*}$ still remains non-trivial. In addition, it is not energy efficient to sense most channels every time slot if there are only a small amount of sensors having data transmission requests. Thus, we propose an active elimination algorithm (AEA) denoted by $\rho_{A E A}$ that can find the $m$ best channels in $\mathcal{C}$ with a finite time horizon and low error rate.

Without loss of generality, we assume that $\mu$ is ordered and $\mu_{1}>\mu_{2}>\ldots>\mu_{K}$. Let $\mu_{m}=\mu^{*}$. We use $\Delta_{i, j}=\mu_{i}-\mu_{j}$ to denote the gap between $\mu_{i}$ and $\mu_{j}$. For the $m$ best channels in $\mathcal{C}$ we define $\Delta_{i}^{m}$ as

$$
\Delta_{i}^{m}=\left\{\begin{array}{lc}
\Delta_{i, m+1}, & 1 \leq i \leq m \\
\Delta_{m, i}, & m+1 \leq i \leq K .
\end{array}\right.
$$

At round $i$, the probability that $C_{n} \in C_{G}$ is eliminated is at most $6 \exp \left(-\frac{\left(\Delta_{m}^{m}\right)^{2}}{2} t\right)$ where $t=\left\lfloor\frac{N}{\left\lceil\log _{2}\left(\frac{K}{m}\right)\right\rceil}\right\rfloor$. AEA is thus an $\varepsilon$-PAC algorithm that can correctly identify the $m$ best channels with probability at least $1-\varepsilon$ where $\varepsilon=\frac{6 m N}{t} \exp \left(-\frac{\left(\Delta_{m}^{m}\right)^{2}}{2} t\right)$. The proof is omitted due to the space limit.

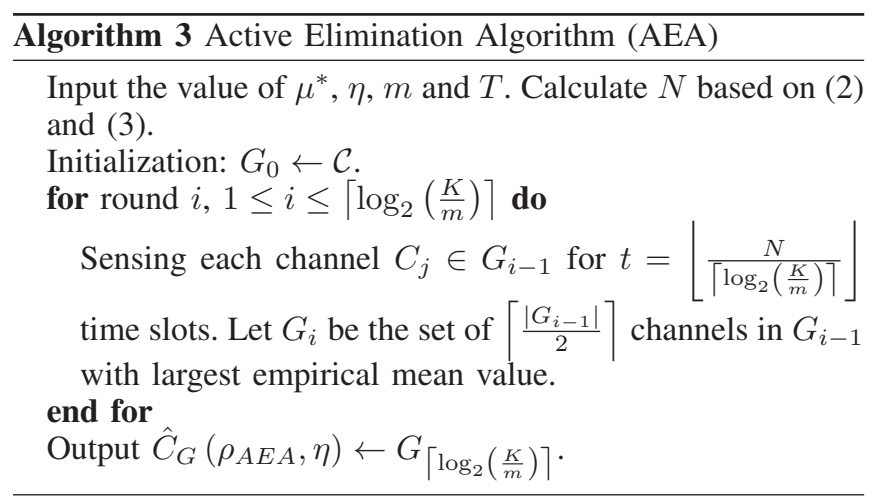

\section{Joint Channel Sensing and Power Control Scheme}

We now propose the joint channel sensing and power control scheme (JCSPCS) to maximize the amount of transmitted data of each sensor. With channel availability constraints and data transmission requests, we use the AEA to find the $m$ best channels for $n$ sensors $(n \leq m \leq K)$ to regularly transmit their data. Let $\varepsilon^{*}$ be the maximum acceptable error rate of AEA. We have $\varepsilon=\frac{6 m N}{t} \exp \left(-\frac{\left(\Delta_{m}^{m}\right)^{2}}{2} t\right) \leq \varepsilon^{*}$. We assume $t=\frac{N}{\log _{2}\left(\frac{K}{m}\right)}$ for simplicity. Thus, we have

$$
N \geq \frac{2 \log _{2}\left(\frac{K}{m}\right)}{\left(\Delta_{m}^{m}\right)^{2}} \ln \left(\frac{6 m \log _{2}\left(\frac{K}{m}\right)}{\varepsilon^{*}}\right) .
$$

We also assume that each sensor $S_{i} \in \mathcal{S}$ has the same circuit power consumption $P_{i, c}=P_{c}$ and channel sensing power consumption $P_{i, s}=P_{s}$. According to (2), (3), (5) and (8), the minimum energy ratio for reliable channel sensing denoted as

$$
\eta \geq \frac{2 \alpha T\left(P_{c}+P_{s}\right) \log _{2}\left(\frac{K}{m}\right)}{\left(\Delta_{m}^{m}\right)^{2} E} \ln \left(\frac{6 m \log _{2}\left(\frac{K}{m}\right)}{\varepsilon^{*}}\right)=\eta^{*}
$$

From (5), we know that $\frac{\left(1-\eta^{*}\right) E}{P_{j}+P_{c}}$ is bounded and inversely proportional to $P_{j}$ while $\log _{2}\left(1+P_{j} g_{i, k}\right)$ is bounded and logarithmically proportional to $P_{j}$. According to the extreme value theorem, for each $S_{i}$ transmitting data on $C_{k}$, there exists a $P_{i, k}^{*}$ that maximizes the total number of transmitted bits.

We propose the joint channel sensing and power control scheme (JCSPCS) shown in Algorithm 4.

\section{Algorithm 4 Joint Channel Sensing and Power Control} Scheme

Input the value of $n, m, K, T, \Delta_{m}^{m}$ and $\varepsilon^{*}$

Initialization: Find the $m$ best channels using AEA. Calculate $\eta^{*}$ according to (9).

for $1 \leq i \leq n$ do

for $1 \leq k \leq m$ do

Calculate $P_{i, k}^{*}$ and $L_{i, k}^{*}$ based on (5). $L \leftarrow L_{i, k}^{*}$.

\section{end for}

end for

Solve (6) using the Hungarian algorithm [6].

Output $\lambda^{*}$ and $L^{*}$, where $\lambda^{*}$ is the $n \times m$ matrix with element $\lambda_{i, k}$ that maximizes $L^{*}$ in (6). 


\section{Simulation Results}

In our simulation, we assume that the channels are slow fading channels. The channel state information (CSI) is available to the transmitter and receiver. The transmission power ranges from 0 to $100 \mathrm{~mW}$ and is equally divided into $M=10$ levels. For channel $C_{k}$ assigned to sensor $S_{i}$, the normalized channel gain $g_{i, k}$ is randomly generated. Some parameters in the simulation are given in TABLE I.

\section{TABLE I: Parameters for simulation}

\begin{tabular}{|l|l|l|}
\hline Symbol & Description & Value \\
\hline$P_{c}$ & circuit power consumption & $10 \mathrm{~mW}$ \\
\hline$P_{s}$ & sensing power consumption & $30 \mathrm{~mW}$ \\
\hline$T$ & length of each time slot & $1 \mathrm{~s}$ \\
\hline$\alpha$ & fraction of time for channel sensing & 0.1 \\
\hline$E$ & total energy & $500 \mathrm{~J}$ \\
\hline$W$ & channel bandwidth & $200 \mathrm{kHz}$ \\
\hline$M$ & transmission power levels & 10 \\
\hline$R^{*}$ & minimum data rate requirement & $100 \mathrm{kbps}$ \\
\hline
\end{tabular}
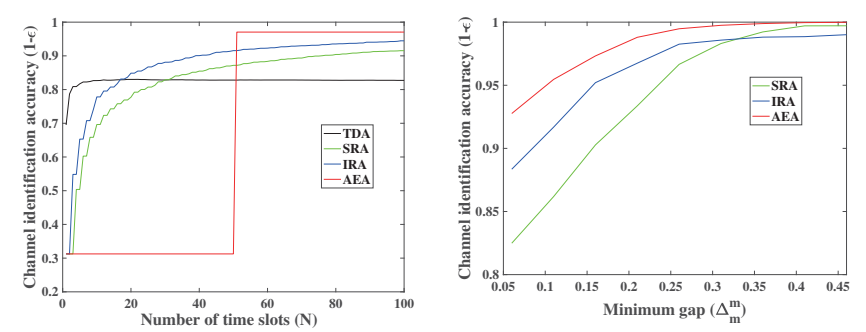

(a) CIA with different channel sens-(b) CIA with different minimum ing algorithms.

channel availability gap $\left(\Delta_{m}^{m}\right)$.

Fig. 2: Channel identification accuracy (CIA)

We first consider the channel sensing problem with availability constraints. We set $K=16, m=5, N=100$, and $\mu^{*}=0.7$. Elements in $\mu=\left\{\mu_{1}, \mu_{2}, \ldots, \mu_{K}\right\}$ are ordered and equally spaced in the interval [0.05,0.95]. In the beginning, all channels are assumed to be good channels. We compared our algorithms with a threshold detection algorithm (TDA). In TDA, we say a channel $C_{i}$ is bad if in any time slot $T_{n}$ we have $\hat{\mu}_{i, n}<\mu^{*}$. The performance of algorithms is averaged over 1000 simulation runs. Fig. 2a shows that all our algorithms correctly identify the good channels with higher accuracy (above $90 \%$ ) compared to that of TDA $(82 \%)$. For the final error rate, we have $\varepsilon\left(\rho_{S R A}, \eta\right)>\varepsilon\left(\rho_{I R A}, \eta\right)>\varepsilon\left(\rho_{A E A}, \eta\right)$. Unlike SRA and IRA that gradually identify bad channels with high confidence, AEA achieves the lowest error rate as it keeps sensing channels until it has high confidence to eliminate at most half of the existing channels. We then investigate how $\Delta_{i, j}$ affects the error rate $\varepsilon$ for our channel sensing algorithms. According to the definition in (7), we have $\Delta_{m}^{m}=\Delta_{m+1}^{m}=\min _{i} \Delta_{i}^{m}$. We set $K=16, m=5, N=30$, and $\mu^{*}=0.7$. We gradually change $\Delta_{m}^{m}$ from 0.05 to 0.45 . We compare the error rates of our algorithms with different values of $\Delta_{m}^{m}$. From Fig. 2b, we show that for all algorithms the error rates $\varepsilon$ decrease with increasing $\Delta_{m}^{m}$. Under the same parameter settings, AEA always has the lowest error rate.

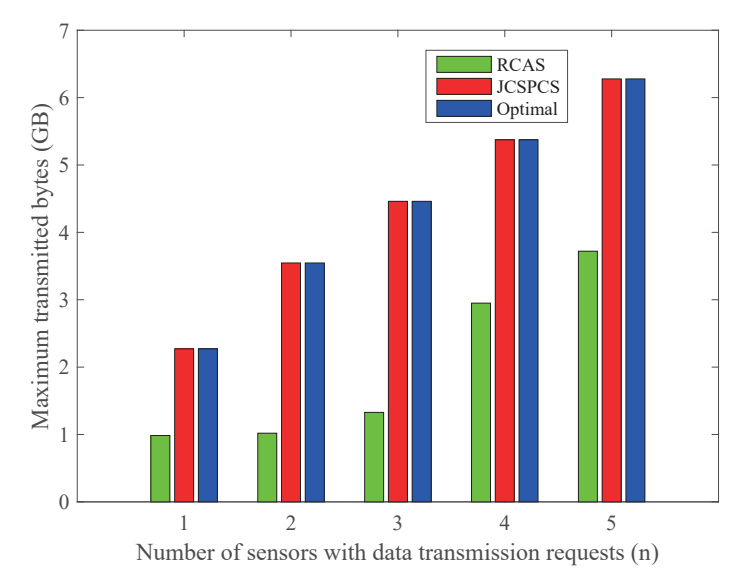

Fig. 3: Maximum number of transmitted bits in the cluster vs. different number of sensors with data transmission requests.

Finally, we show the efficiency of JCSPCS in Fig. 3. Here, we investigate JCSPCS with different $n$. We set $K=16$, $m=5, \varepsilon^{*}=0.1, \Delta_{m}^{m}=0.06$ and $\mu^{*}=0.7$. Fig. 3 shows that JCSPCS outperforms the random channel access scheme (RCAS) with different $n$ in our simulation and JCSPCS is the optimal solution. Note that Hungarian algorithm [6] has $O\left(n^{4}\right)$ time complexity. However, this is not a problem in practice since the number of sensors with data transmission requests in each cluster is small $\left(n \sim 10^{1}\right)$.

\section{CONCLUSION}

In this paper, we considered power control and channel sensing problem in a CRWSN. We proposed three $\varepsilon$-PAC algorithms to solve the channel sensing problem with availability constraints. Then we considered the power control problem with QoS constraints and aimed at maximizing the total number of transmitted bits in the cluster. A joint channel sensing and power control scheme (JCSPCS) is proposed to optimize the total data transmission. Simulation results showed that our $\varepsilon$-PAC algorithms can correctly identify good channels with low error rate. We also showed that JCSPCS maximized the number of transmitted bits in the cluster.

\section{REFERENCES}

[1] M. McHenry, E. Livsics, T. Nguyen, and N. Majumdar, "Xg dynamic spectrum access field test results [topics in radio communications]," IEEE Communications Magazine, vol. 45, no. 6, pp. 51-57, June 2007.

[2] O. B. Akan, O. B. Karli, and O. Ergul, "Cognitive radio sensor networks," Netwrk. Mag. of Global Internetwkg., vol. 23, no. 4, pp. 34-40, Jul. 2009. [Online]. Available: http://dx.doi.org/10.1109/MNET.2009.5191144

[3] P. Auer, N. Cesa-Bianchi, and P. Fischer, "Finite-time analysis of the multiarmed bandit problem," Machine learning, vol. 47, no. 2-3, pp. 235-256, 2002.

[4] C. Tekin and M. Liu, "Online learning in opportunistic spectrum access: A restless bandit approach," in INFOCOM, 2011 Proceedings IEEE, April 2011, pp. 2462-2470.

[5] Y. Gai and B. Krishnamachari, "Decentralized online learning algorithms for opportunistic spectrum access," in Global Telecommunications Conference (GLOBECOM 2011), 2011 IEEE. IEEE, 2011, pp. 1-6.

[6] H. W. Kuhn, "The hungarian method for the assignment problem," Naval Research Logistics Quarterly, vol. 2, pp. 83-97, 1955. 